\title{
Switching the magnetostructural coupling in MnCoGe-based magnetocaloric materials
}

\author{
Xuefei Miao $\odot,{ }^{1, *}$ Yong Gong, ${ }^{1}$ Luana Caron,,${ }^{2} \dagger$ Yurong You, ${ }^{1}$ Guizhou Xu,${ }^{1}$ Denis Sheptyakov $\odot,{ }^{3}$ Pascal Manuel, ${ }^{4}$ \\ Fengjiao Qian, ${ }^{5}$ Yujing Zhang, ${ }^{1}$ Feng Xu, ${ }^{1,}$ Niels van Dijk, ${ }^{6}$ and Ekkes Brück ${ }^{6}$ \\ ${ }^{1}$ MIIT Key Laboratory of Advanced Metallic and Intermetallic Materials Technology, School of Materials Science and Engineering, \\ Nanjing University of Science and Technology, 210094 Nanjing, China \\ ${ }^{2}$ Department of Physics, Bielefeld University, 33501 Bielefeld, Germany \\ ${ }^{3}$ Laboratory for Neutron Scattering and Imaging, Paul Scherrer Institut, 5232 Villigen, Switzerland \\ ${ }^{4}$ ISIS facility, Rutherford Appleton Laboratory, Chilton, Didcot, Oxfordshire OX11 OQX, United Kingdom \\ ${ }^{5}$ College of Physics, Nanjing University of Aeronautics and Astronautics, 210016 Nanjing, China \\ ${ }^{6}$ Fundamental Aspects of Materials and Energy, Department of Radiation Science and Technology, Delft University of Technology, \\ Mekelweg 15, 2629 JB Delft, The Netherlands
}

(Received 27 July 2020; revised 22 September 2020; accepted 25 September 2020; published 14 October 2020)

\begin{abstract}
We performed neutron-diffraction experiments and density functional theory calculations to study the magnetostructural coupling in $\mathrm{MnCoGeB}_{x}(x=0,0.01$, and 0.05$)$ alloys. By varying the amount of boron addition, we are able to freely switch the magnetostructural coupling on and off in the MnCoGe alloys. It is found that the boron addition stabilizes the high-temperature hexagonal phase due to the reduced interatomic distances and the enhanced covalent bonding. The hexagonal-orthorhombic structural transition shifts to low temperatures with the boron addition and coincides with the paramagnetic-ferromagnetic (PM-FM) transition in the $\mathrm{MnCoGeB}_{0.01}$ alloy. With a further increase in the boron addition, the structural and magnetic transitions are decoupled again. The hexagonal-orthorhombic structural transition is significantly suppressed in the $\mathrm{MnCoGeB}_{0.05}$ alloy, although subtle distortions in the hexagonal structure are evidenced by a canted spin arrangement below $75 \mathrm{~K}$. The MnCoGe and $\mathrm{MnCoGeB}_{0.01}$ alloys show a collinear FM structure, having a much larger Mn moment than the $\mathrm{MnCoGeB}_{0.05}$ alloy. The relatively small $\mathrm{Mn}$ moment in the $\mathrm{MnCoGeB}_{0.05}$ alloy can be attributed to the shortened Mn-Mn distance and the enhanced overlap of the $3 d$ orbitals between the neighboring Mn atoms. The uncovered relationship between the structural evolution and the sizable magnetic moment in the present work offers more insight into the magnetostructural coupling in the MnCoGe-based alloys.
\end{abstract}

DOI: 10.1103/PhysRevMaterials.4.104407

\section{INTRODUCTION}

Magnetic refrigeration, demonstrating a high energy efficiency and low environmental impact, has a great potential to replace conventional vapor-compression refrigeration [1-3]. Magnetic refrigeration takes advantage of the magnetocaloric effect (MCE) [4], which is manifested by an adiabatic temperature change $\left(\Delta T_{\mathrm{ad}}\right)$ and an isothermal entropy change $\left(\Delta S_{\text {iso }}\right)$ of a magnetic material in response to a driving magnetic field. Although the MCE is intrinsic to all magnetic materials, it is most pronounced (the so-called giant MCE) in those exhibiting a first-order magnetic transition (FOMT) due to the presence of latent heat.

In strong contrast to the conventional second-order magnetic transition, the FOMT is characteristic of the coincidence of a magnetic and a structural transition. Therefore, the search for promising magnetocaloric materials can be carried out either by screening the magnetic materials with a naturally coupled magnetic and structural transition, or by tailoring

\footnotetext{
*Corresponding author: xuefeimiao@njust.edu.cn

†Corresponding author: luana.caron@uni-bielefeld.de

‡Corresponding author: xufeng@njust.edu.cn
}

the naturally separated magnetic and structural transitions to coincide. The ( $\mathrm{Mn}, \mathrm{Fe})_{2}(\mathrm{P}, \mathrm{Si})[5-7]$ and $\mathrm{La}(\mathrm{Fe}, \mathrm{Si})_{13}[8,9]$ materials are typical examples for the former, and the latter is well demonstrated in the MnCoGe alloys.

The stoichiometric MnCoGe alloy undergoes a martensitic transition from the high-temperature $\mathrm{Ni}_{2}$ In-type hexagonal (hex) to the low-temperature TiNiSi-type orthorhombic (ort) structure at the transition temperature $\left(T_{t}\right)$ of around $430 \mathrm{~K}$ [10]. The orthorhombic martensite shows a second-order ferromagnetic $(\mathrm{FM})$ transition at the Curie temperature $\left(T_{C}{ }^{\text {ort }}\right)$ of about $345 \mathrm{~K}$, which is below the $T_{t}$ [10]. Metastable hexagonal austenite can be obtained via quenching the sample from high temperatures, which also shows a second-order FM transition with a Curie temperature $\left(T_{C}^{\text {hex }}\right)$ of around $283 \mathrm{~K}$ [11]. Apparently, the magnetic and structural transitions are separated in the stoichiometric MnCoGe alloy. Lots of effort has been made to manipulate the magnetic and structural transitions in order to achieve a coupled magnetic and structural transition, i.e. a magnetostructural transition. The introduction of vacancies $[12,13]$ and the design of off-stoichiometric compositions [14] both enable the coincidence of the magnetic and structural transitions. Besides that, the partial replacement of the $\mathrm{Mn}$ or Co atoms by some $3 d$ transition metal elements [15-21], as well as the substitution of the Ge by In [22] or 
Si [23] can also realize a magnetostructural transition in the MnCoGe alloys. Additionally, hydrostatic pressure offers an alternative approach to tailor the magnetostructural coupling in the MnCoGe-type alloys [24-27].

The coincidence of the magnetic and structural transitions in the MnCoGe alloys via applying external pressure or tuning the composition can phenomenologically be attributed to a rapid decrease in $T_{t}$. The underlying mechanism is however still unclear. For the compositions in which the hexagonalorthorhombic structural transition is suppressed completely, the hexagonal phase exhibits a much smaller saturation magnetic moment than the orthorhombic phase [11]. This suggests significant changes in the magnetic exchange coupling accompanying the hexagonal-orthorhombic structural transition. Therefore, further studies are required to uncover the relationship between the structural evolution and the sizable magnetic moment during the FOMT of the MnCoGe-based alloys.

In the present study, we performed temperature-dependent neutron diffraction (ND) experiments and density functional theory (DFT) calculations on the $\operatorname{MnCoGeB}_{x}(x=0,0.01$, and 0.05 ) alloys. By varying the amount of boron addition, we are able to freely switch the coupling between the magnetic and structural transitions on and off. This allows us to study the different magnetic structures and phase-transition behaviors in the MnCoGe-based alloys, which sheds more light on the mechanism of their magnetostructural coupling.

\section{EXPERIMENTAL DETAILS}

\section{A. Synthesis}

Polycrystalline $\mathrm{MnCoGeB}_{x}(x=0,0.01$, and 0.05) alloys were prepared by arc-melting appropriate amounts of highpurity elemental starting materials under Ar atmosphere in a water-cooled copper crucible. It should be noted that the ${ }^{11} \mathrm{~B}$ isotope was used as starting material to avoid the strong absorption of neutrons by the ${ }^{10} \mathrm{~B}$ isotopes in natural $\mathrm{B}$. The ingots were sealed in quartz ampoules under Ar atmosphere, annealed at $1123 \mathrm{~K}$ for $120 \mathrm{~h}$, and then slowly cooled down to room temperature.

\section{B. Neutron diffraction and magnetic characterization}

Temperature-dependent neutron-diffraction experiments on the $\mathrm{MnCoGe}$ and $\mathrm{MnCoGeB}_{0.01}$ alloys were performed on the high-resolution powder diffractometer (HRPT) at Paul Scherrer Institut in Switzerland [28]. The wavelength of the neutrons was $1.8857 \AA$. The neutron-diffraction measurements on the $\mathrm{MnCoGeB}_{0.05}$ sample were carried out on the WISH time-of-flight diffractometer at the ISIS Facility, Rutherford Appleton Laboratory in the UK [29]. The neutron-diffraction data were refined using FULLPROF's implementation of the Rietveld method [30]. Magnetic properties were characterized using a superconducting quantum interference device magnetometer (MPMS XL, Quantum Design).

\section{COMPUTATIONAL DETAILS}

The electronic structure calculations for the $\mathrm{MnCoGeB}_{x}$ $(x=0,0.01$, and 0.05$)$ alloys were carried out using the CASTEP code based on the density functional theory [31].
The unit-cell parameters (e.g., lattice parameters, atomic coordinates, and occupancy) derived from ND experiments at 1.2 and $1.5 \mathrm{~K}$ were used as input for the DFT calculations. For simplicity of the DFT calculation, we assumed collinear FM structures for both orthorhombic and the hexagonal phases. Additionally, the boron atoms (less than 5 at. \%) were not considered in the orthorhombic or hexagonal structure models in the calculations. The exchange-correlation functions were treated with generalized gradient approximation (GGA) in the parametrization of PW91 [32]. An energy cutoff of $500 \mathrm{eV}$ was applied for the plane-wave function and a $k$-point mesh of $13 \times 13 \times 12$ was selected for the Brillouin-zone integration.

Additionally, the electron localization function (ELF) [33] of the MnCoGe-based alloys was calculated in the present work. The ELF has been widely used to describe and visualize chemical bonds in molecules and solids, indicating the electron-pair distribution in terms of interatomic bonding [34-38]. The ELF introduced by Becke and Edgecombe can be described as [33]

$$
\mathrm{ELF}=1 /\left[1+\chi^{2}(\boldsymbol{r})\right]
$$

and

$$
\chi(\boldsymbol{r})=D(\boldsymbol{r}) / D_{h}(\boldsymbol{r}),
$$

where $D(\boldsymbol{r})$ is the curvature of the spherically averaged probability density to find another same-spin electron around a reference electron located at position $\boldsymbol{r}$ and $D_{h}(\boldsymbol{r})$ corresponds to a uniform electron gas of the same electron density. The $\chi(\boldsymbol{r})$ is thus a dimensionless localization index calibrated with respect to the uniform-density electron gas as reference. The ELF value is between 0 and 1 . The value ELF $=1$ at the specific position corresponds to a complete electron pair localization, indicating a strong covalent bonding, and the value ELF $=0$ denotes the borders between electron pairs. The topological analysis of the ELF provides visualized information on the type and strength of chemical bonds. The unit-cell parameters at 1.2 and $1.5 \mathrm{~K}$ derived from the ND experiments were used for the ELF calculations.

\section{RESULTS AND DISCUSSION}

\section{A. Realization of a magnetostructural FOMT}

The magnetization $(M)$ as a function of temperature $(T)$ for the $\mathrm{MnCoGeB}_{x}(x=0,0.01$, and 0.05) alloys is shown in Fig. 1(a). A continuous magnetic transition occurs at around $345 \mathrm{~K}$ in the parent MnCoGe alloy, which corresponds to the FM transition of the orthorhombic phase [10]. With a small amount of boron addition $(x=0.01)$ a sharp FM transition appears with noticeable thermal hysteresis. This suggests the realization of a magnetostructural FOMT in the $\mathrm{MnCoGeB}_{0.01}$ alloy. With a further increase in the boron addition to $x=$ 0.05 , the magnetic transition changes back to a typical secondorder FM transition with a $T_{C}$ around $260 \mathrm{~K}$.

Figure 1(b) plots the isothermal magnetization curves obtained at $5 \mathrm{~K}$ for the $\mathrm{MnCoGeB}_{x}$ alloys. All the samples display a soft FM behavior. The saturation magnetic moment 

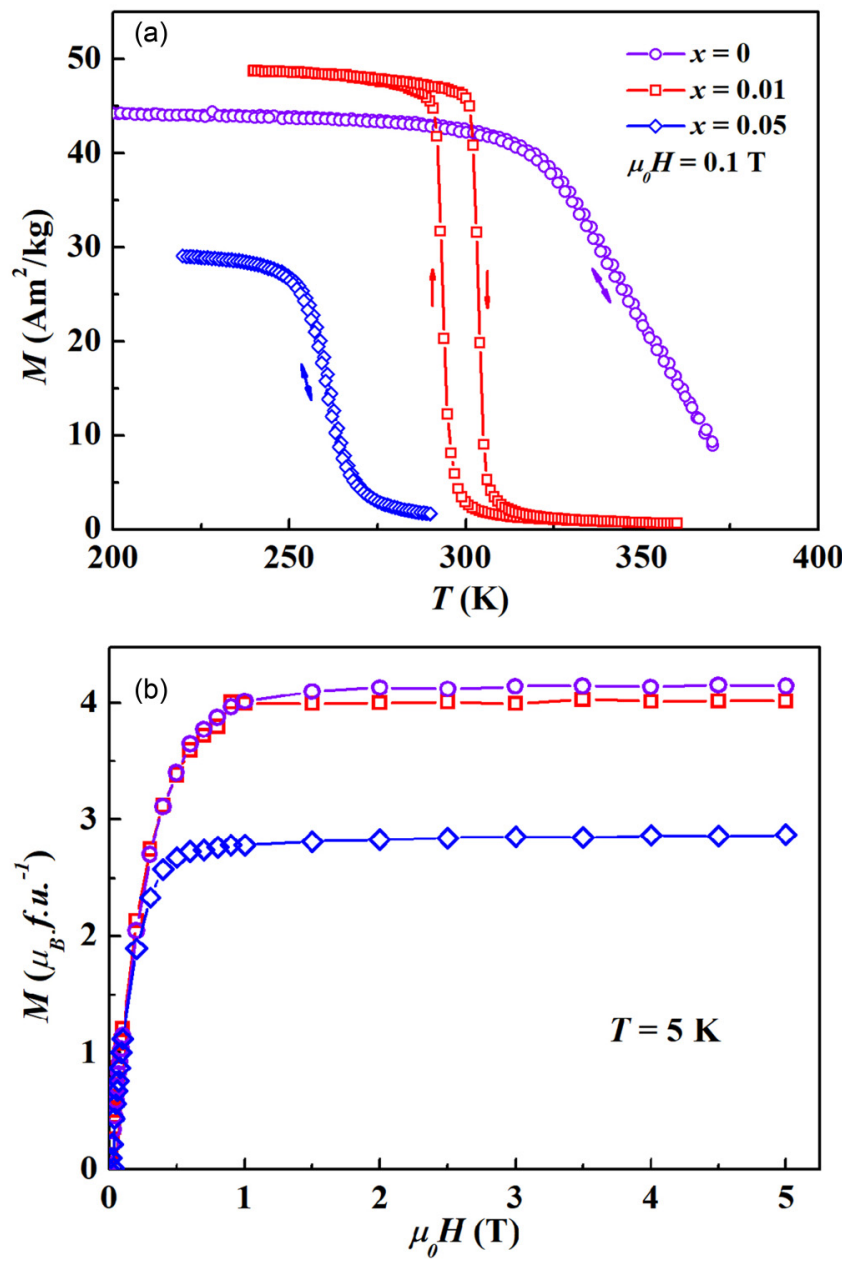

FIG. 1. Magnetization as a function of temperature (a) and external magnetic field (b) for the $\operatorname{MnCoGeB}_{x}(x=0,0.01$, and 0.05) alloys.

$\left(M_{S}\right)$ at $5 \mathrm{~K}$ is significantly reduced in the $\mathrm{MnCoGeB}_{0.05}$ alloy in comparison with the other two alloys.

Figure 2 shows the contour plots of the ND patterns measured at different temperatures for the $\mathrm{MnCoGeB}_{x}(x=0$, 0.01 , and 0.05 ) alloys. For conciseness and clarity, only the regions near the transition temperatures are presented. As shown in Fig. 2(a), the MnCoGe parent alloy stays in the TiNiSi-type orthorhombic structure (space group Pnma, no. 62) within our measurement temperature range (from 1.2 to $450 \mathrm{~K})$. The low- $Q$ peak intensity is slightly reduced above $345 \mathrm{~K}$, due to the disappearing magnetic diffraction contribution upon a transition to the paramagnetic (PM) state. For the $\mathrm{MnCoGeB}_{0.01}$ alloy, markedly distinct diffraction patterns can be observed at high and low temperatures, as shown in Fig. 2(b). The ND patterns above $310 \mathrm{~K}$ can be refined with purely nuclear scattering from the $\mathrm{Ni}_{2} \mathrm{In}$-type hexagonal structure (space group $\mathrm{Pb}_{3} / \mathrm{mmc}$, no. 194). In strong contrast to the high-temperature patterns, the ND patterns below 295 $\mathrm{K}$ can be refined with both nuclear and magnetic contributions from the TiNiSi-type orthorhombic structure. The ND patterns collected between 295 and $310 \mathrm{~K}$ correspond to a superposition of the high- and low-temperature ND patterns. Therefore, the temperature-dependent ND patterns clearly demonstrate a
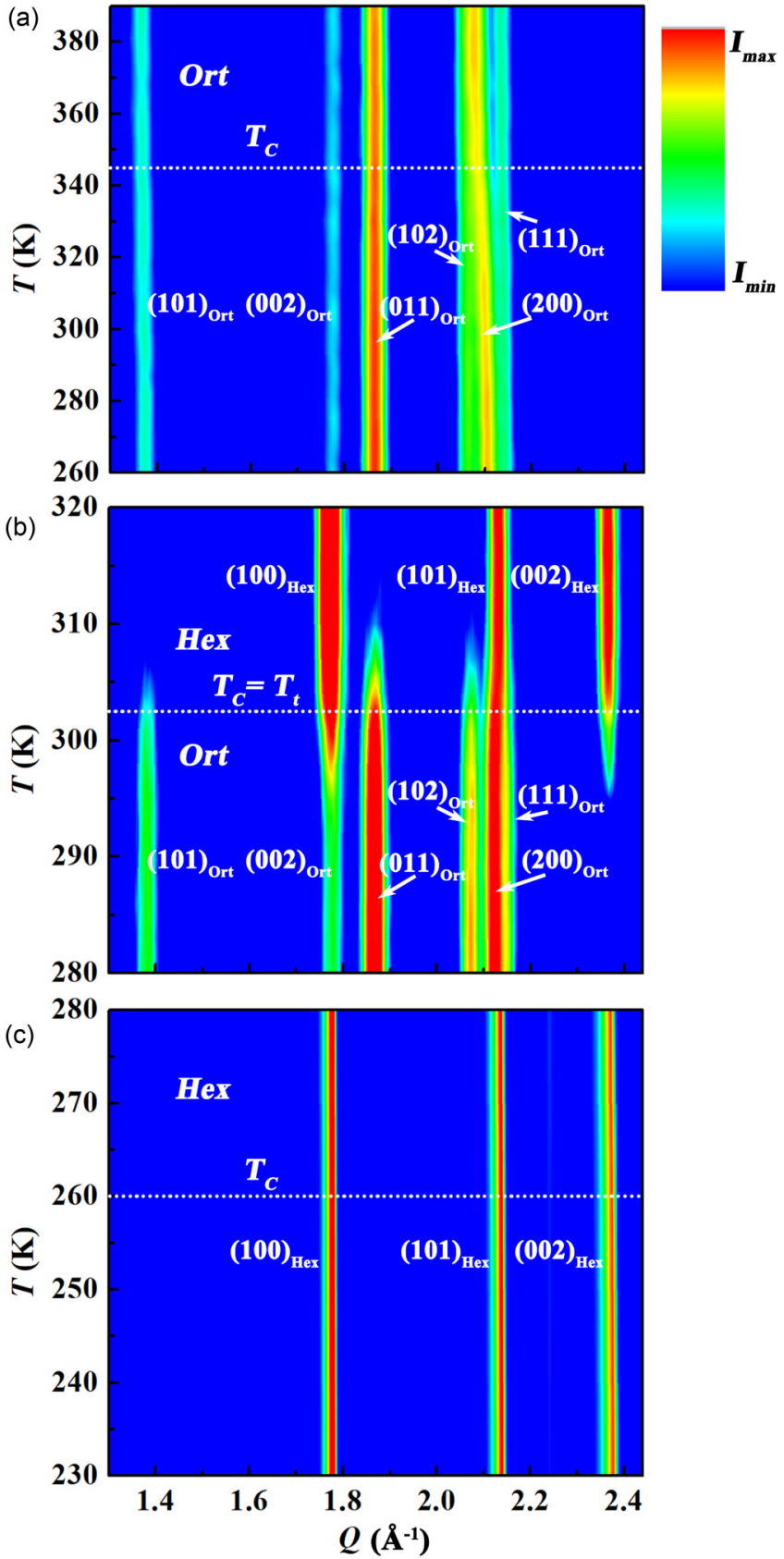

FIG. 2. Contour plots of the temperature-dependent neutrondiffraction patterns for $\mathrm{MnCoGeB}_{x}$ with $x=0$ (a), 0.01 (b), and 0.05 (c). The scale bar on the right represents the normalized intensity. The white dotted lines in all panels represent the magnetic transition temperatures.

magnetostructural FOMT from the FM orthorhombic phase to the PM hexagonal phase in the $\mathrm{MnCoGeB}_{0.01}$ alloy. When the boron content is further increased to $x=0.05$, the hexagonal $\mathrm{Ni}_{2}$ In-type structure is retained during the FM-PM transition around $260 \mathrm{~K}$. This indicates the decoupling of the magnetic and structural transitions in the $\mathrm{MnCoGeB}_{0.05}$ alloy. Consequently, the magnetic and ND experiments reveal that boron addition offers an efficient and effective way to manipulate the magnetostructural coupling in the MnCoGe alloys. 
TABLE I. Structural and magnetic parameters derived from neutron diffraction, DFT calculation, and magnetometry measurements for the $\mathrm{MnCoGeB}_{x}$ alloys.

\begin{tabular}{|c|c|c|c|}
\hline $\begin{array}{l}T(\mathrm{~K}) \\
\text { Magnetic space group }\end{array}$ & $\begin{array}{c}\mathrm{MnCoGe} \\
1.2 \\
P n^{\prime} m^{\prime} a(\text { no. 62.446) }\end{array}$ & $\begin{array}{c}\mathrm{MnCoGeB}_{0.01} \\
1.2 \\
P n^{\prime} m^{\prime} a(\text { no. 62.446) }\end{array}$ & $\begin{array}{c}\mathrm{MnCoGeB}_{0.05} \\
1.5 \\
\text { Pnm' }^{\prime} a^{\prime}(\text { no. 62.447) }\end{array}$ \\
\hline$a(\AA)$ & $5.9192(1)$ & $5.8821(1)$ & $5.2579(1)$ \\
\hline$b(\AA)$ & $3.8132(1)$ & $3.8094(1)$ & $4.0697(1)$ \\
\hline$c(\AA)$ & $7.0621(1)$ & $7.0415(1)$ & $7.0529(2)$ \\
\hline$V\left(\AA^{3}\right)$ & $159.399(4)$ & $157.783(4)$ & $150.920(4)$ \\
\hline$d_{\mathrm{Mn}-\mathrm{Mn}}(\AA)$ & $3.079(2)$ & $3.064(3)$ & $2.629(1)$ \\
\hline$d_{\mathrm{Mn}-\mathrm{Co}}(\AA)$ & $2.736(3)$ & $2.728(4)$ & $2.683(6)$ \\
\hline$d_{\mathrm{Mn}-\mathrm{Ge}}(\AA)$ & $2.614(1)$ & $2.596(2)$ & $2.683(6)$ \\
\hline$d_{\mathrm{Co}-\mathrm{Co}}(\AA)$ & $2.813(3)$ & $2.799(4)$ & $3.519(3)$ \\
\hline$d_{\mathrm{Co}-\mathrm{Ge}}(\AA)$ & $2.337(3)$ & $2.318(2)$ & $2.339(5)$ \\
\hline$\mu_{\mathrm{Mn}}\left(\mu_{\mathrm{B}}\right)$ from ND & $3.54(3)$ & $3.08(4)$ & $2.20(3)$ \\
\hline$\mu_{\mathrm{Mn}}\left(\mu_{\mathrm{B}}\right)$ from $\mathrm{DFT}$ & 3.64 & 3.35 & 2.18 \\
\hline$\mu_{\mathrm{Co}}$ from ND & $0.63(3)$ & $0.81(5)$ & $0.76(4)$ \\
\hline$\mu_{\mathrm{Co}}\left(\mu_{\mathrm{B}}\right)$ from $\mathrm{DFT}$ & 0.67 & 0.65 & 0.60 \\
\hline $\mathrm{M}_{\mathrm{s}}\left(\mu_{\mathrm{B}}\right.$ f.u. $\left.^{-1}\right)$ from MPMS & 4.16 & 4.02 & 2.87 \\
\hline$R_{p}(\%)$ & 4.29 & 5.63 & 5.59 \\
\hline$R_{\text {Bragg }}(\%)$ & 4.18 & 5.66 & 3.21 \\
\hline
\end{tabular}

\section{B. Magnetic structure of the $\mathrm{MnCoGeB}_{x}$ alloys}

We further performed a symmetry analysis and Rietveld refinement on the low-temperature ND data to determine the magnetic structure of the $\mathrm{MnCoGeB}_{x}$ alloys.

For the $\mathrm{MnCoGe}$ and $\mathrm{MnCoGeB}_{0.01}$ alloys, all the diffraction peaks observed at $1.2 \mathrm{~K}$ can be indexed with a TiNiSi-type orthorhombic structure (space group Pnma, no. 62). This suggests a parent space group of Pnma (no. 62) with a propagation vector $\boldsymbol{k}=(0,0,0)$ for the magnetic structure. Symmetry analysis was performed to calculate the possible Shubnikov magnetic space groups using the online program MAXMAGN [39] in the Bilbao Crystallographic server. We obtained eight possible Shubnikov magnetic space groups allowing nonzero magnetic moments for the parent space group Pnma (no. 62) with a propagation vector $\boldsymbol{k}=(0,0,0):$ Pnma (no. 62.441), $P n^{\prime} m a$ (no. 62.443), $P n m^{\prime} a$ (no. 62.444), $P n m a^{\prime}$ (no. 62.445), $P n^{\prime} m^{\prime} a$ (no. 62.446), $P n m^{\prime} a^{\prime}$ (no. 62.447), $P n^{\prime} m a^{\prime}$ (no. 62.448) and $P n^{\prime} m^{\prime} a^{\prime}$ (no. 62.449). The direction and size of the Mn and Co moments in the eight possible magnetic space groups are summarized in Table SI of the Supplemental Material [40].

We carefully checked all the possible Shubnikov magnetic space groups in the refinements and found that only $P n^{\prime} m^{\prime} a$ (no. 62.446) provides the solution to the magnetic structure of the $\mathrm{MnCoGe}$ and $\mathrm{MnCoGeB}_{0.01}$ alloys at $1.2 \mathrm{~K}$. Figures 3(a) and 3(b) show the corresponding Rietveld refinements of the ND patterns collected at $1.2 \mathrm{~K}$ for the $\mathrm{MnCoGe}$ and $\mathrm{MnCoGeB}_{0.01}$ alloys, respectively. The refinement results are summarized in Table I. A schematic representation of the corresponding magnetic structure is illustrated in Fig. 3(c). In this ferromagnetic structure, both the Mn and Co moments are aligned along the $c$ axis of the orthorhombic structure. The Mn and Co moments are 3.54(3) $\mu_{\mathrm{B}}$ and $0.63(3) \mu_{\mathrm{B}}$, respectively in the $\mathrm{MnCoGe}$ alloy, while they are 3.08(4) $\mu_{B}$ and $0.81(5) \mu_{\mathrm{B}}$, respectively in the $\mathrm{MnCoGeB}_{0.01}$ alloy. We also performed DFT calculations on the magnetic structure of the $\mathrm{MnCoGeB}_{x}$ alloys. The unit-cell parameters at $1.2 \mathrm{~K}$ derived from the ND patterns were used as input for the DFT calculations (see Sec. III for details). The calculated magnetic moments are close to those obtained from the ND experiments (see Table I). The MnCoGe alloy shows a decrease in the Mn moment upon a small amount (1 at. \%) of boron addition. This may be due to the enhanced overlap of the $3 d$ orbitals since the $\mathrm{Mn}-\mathrm{Mn}$ and $\mathrm{Mn}$-Co interatomic distances are shortened by the boron addition, as summarized in Table I. The Co moment shows less variation with the boron addition compared to the Mn moment.

Additionally, the contraction of the orthorhombic lattice upon the addition of boron atoms, having a smaller atomic radius than the $\mathrm{Mn}$ and $\mathrm{Co}$ atoms, clearly indicates that the boron atoms have entered the crystal lattice and substituted part of the Mn, Co, or Ge atoms. The differences in the coherent neutron-scattering length of Mn $(-3.73 \mathrm{fm})$, Co (2.49 $\mathrm{fm}), \mathrm{Ge}(8.185 \mathrm{fm})$, and ${ }^{11} \mathrm{~B}(6.65 \mathrm{fm})$ could potentially have allowed for the determination of the preferential site for the boron atoms. Our attempts to localize the preferential site for the boron atoms from the refinements based on the ND data however did not deliver any statistically significant results, probably due to the low boron content $(\leqslant 5$ at. $\%)$. Since the preferential site for the boron atoms is not the primary interest of our paper, we assume that the boron atoms statistically occupy the $\mathrm{Mn}, \mathrm{Co}$, and Ge sites in our refinements.

For the $\mathrm{MnCoGeB}_{0.05}$ alloy, a comparison of the ND pattern collected at 1.5 and $300 \mathrm{~K}$ is shown in Fig. 4(a). At $300 \mathrm{~K}$, all the peaks can be indexed with the Miller indices from the hexagonal space group $P 6_{3} / m m c$ (no. 194). Compared with the pattern obtained at $300 \mathrm{~K}$, the pattern collected at $1.5 \mathrm{~K}$ shows an increased intensity in the (001), (100), (102), (110), and (200) peaks, which suggests the possible space group $P 6_{3} / m m c$ (no. 194) with a propagation vector $\boldsymbol{k}=$ $(0,0,0)$ for the magnetic structure at $1.5 \mathrm{~K}$. Besides that, the increase in the intensity of both the (001) and (100) peaks 

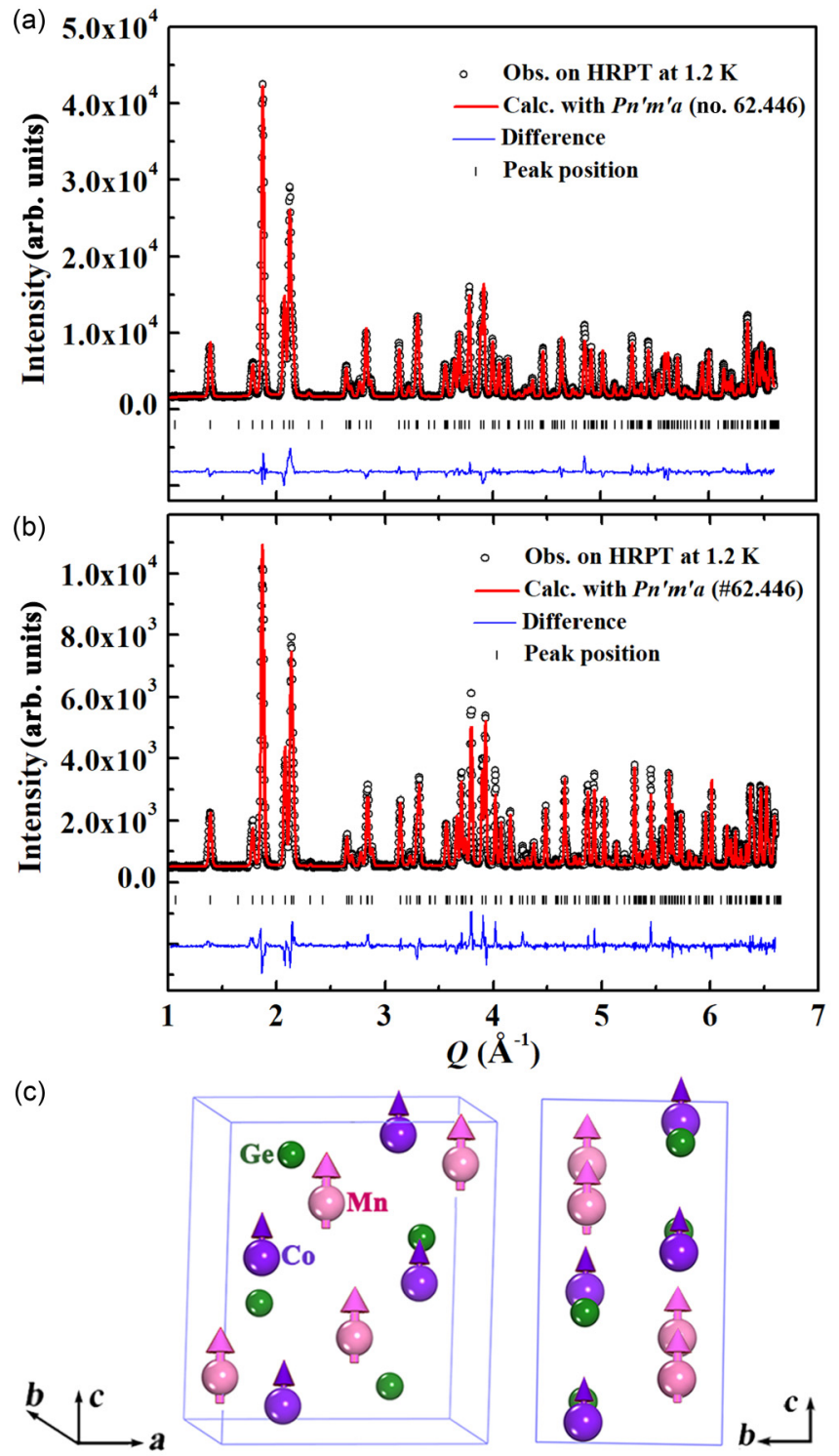

FIG. 3. Rietveld refinement of the ND patterns collected at $T=$ $1.2 \mathrm{~K}$ for (a) the $\mathrm{MnCoGe}$ and (b) $\mathrm{MnCoGeB}_{0.01}$ alloys. Note that the nuclear and magnetic diffraction have the same peak positions. (c) Schematic representation of the magnetic structure in the $\mathrm{MnCoGe}$ and $\mathrm{MnCoGeB}_{0.01}$ alloys at $1.2 \mathrm{~K}$.

indicates a canted spin arrangement in the magnetic structure. The symmetry analysis using the online program MAXMAGN [39] gives three possible Shubnikov magnetic space groups allowing nonzero magnetic moments for the parent space group $P 6_{3} / m m c$ (no. 194) with a propagation vector $\boldsymbol{k}=$ (0, 0, 0): $P 6_{3}{ }^{\prime} / \mathrm{mm}^{\prime} \mathrm{c}$ (no. 194.266), $P 6_{3}{ }^{\prime} / \mathrm{m}^{\prime} \mathrm{m}^{\prime} \mathrm{c}$ (no. 194.268) and $P 6_{3} / \mathrm{mm}^{\prime} \mathrm{c}^{\prime}$ (no. 194.270). However, as shown in Table SII of the Supplemental Material [40], only the $z$ component is allowed for both Mn and Co moments in the three Shubnikov magnetic space groups, which cannot account for the (001) ND peak observed at $1.5 \mathrm{~K}$. Consequently, the hexagonal $\mathrm{P6}_{3} / \mathrm{mmc}$ (no. 194) space group may not be the real parent space group for the magnetic structure of $\mathrm{MnCoGeB}_{0.05}$ alloy at $1.5 \mathrm{~K}$. A similar phenomenon has also been observed in the $\mathrm{MnFeGe}$ alloy, belonging to the same $\mathrm{MnM}^{\prime} \mathrm{Ge}\left(M^{\prime}=\right.$
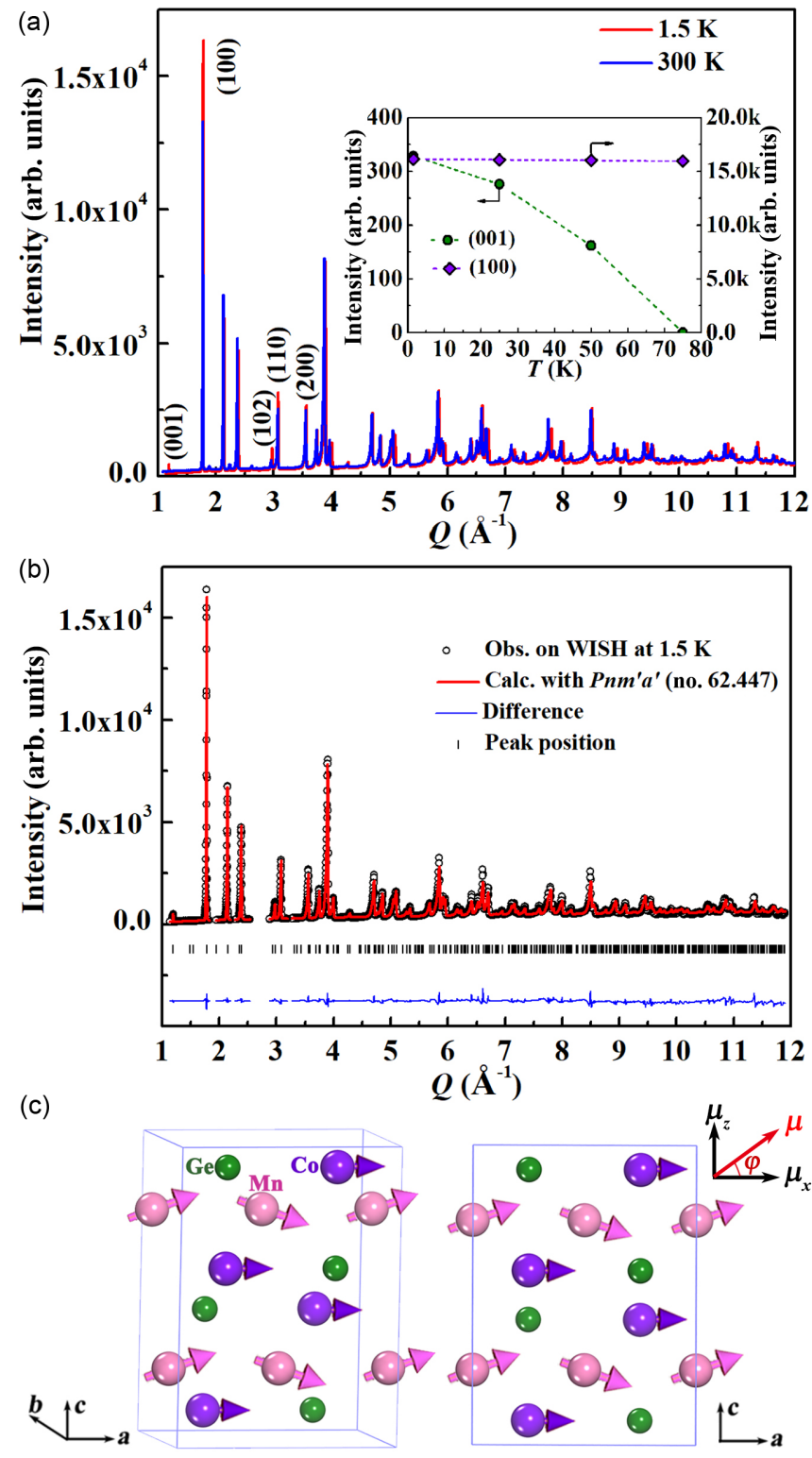

FIG. 4. (a) The ND patterns of the $\mathrm{MnCoGeB}_{0.05}$ alloy collected at 300 and $1.5 \mathrm{~K}$. The intensity of the $(001)$ and (100) peaks as a function of temperature is shown in the inset of (a). (b) Rietveld refinement of the ND patterns collected at $T=1.5 \mathrm{~K}$ for the $\mathrm{MnCoGeB}_{0.05}$ alloy. Note that the nuclear and magnetic diffraction have the same peak positions. Some small peaks from an unknown phase showing in the ND patterns at both 300 and $1.5 \mathrm{~K}$ have been excluded in the refinements. (c) Schematic representation of the magnetic structure in $\mathrm{MnCoGeB}_{0.05}$ alloy at $1.5 \mathrm{~K}$.

transition $3 d$ metals) family as the MnCoGe alloy [41]. ND experiments indicate that $\mathrm{MnFeGe}$ crystallizes in a hexagonal structure (space group $P 6_{3} / m m c$, no. 194) at $300 \mathrm{~K}$ [41]. Upon cooling to $80 \mathrm{~K}$, both (001) and (100) peaks in the ND pattern show increased intensity, suggesting a canted spin arrangement [41], which cannot be described by any magnetic structures derived from the hexagonal $P 6_{3} / m m c$ (no. 194) parent space group.

The $\mathrm{Mn} M / \mathrm{Ge}$ alloys usually crystallize in the $\mathrm{Ni}_{2} \mathrm{In}$ type hexagonal structure $\left(P 6_{3} / m m c\right.$, no. 194) or its distorted 
derivative, the TiNiSi-type orthorhombic structure (Pnma, no. 62) $[42,43]$. These two structures are connected by a diffusionless, displacive martensitic transition, where the unit-cell parameters are related by $a_{\text {ort }}=c_{\text {hex }}, b_{\text {ort }}=a_{\text {hex }}$, and $c_{\text {ort }}=$ $\sqrt{ } 3 a_{\text {hex }}$. Szytuła et al. [41] found that the magnetic structures derived from the orthorhombic Pnma (no. 62) space group allow a canted spin arrangement and can well fit the ND pattern of the MnFeGe alloy collected at $80 \mathrm{~K}$. Due to the crystallographic relationship between the hexagonal and orthorhombic structures, the lattice parameters of the orthorhombic structure are constrained by $c_{\text {ort }}=\sqrt{ } 3 b_{\text {ort }}$ in their refinements [41].

In the present work we also assumed the orthorhombic Pnma (no. 62) parent space group for the magnetic structure of the $\mathrm{MnCoGeB}_{0.05}$ alloy at $1.5 \mathrm{~K}$. The symmetry analysis of the parent space group Pnma (no. 62) with a propagation vector $\boldsymbol{k}=(0,0,0)$ gives eight possible Shubnikov magnetic space groups, as listed in Table S1 of the Supplemental Material [40]. After checking all the possible magnetic space groups in the refinements, we found that only $P n m^{\prime} a^{\prime}$ (no. 62.447) provides the solution to the magnetic structure of the $\mathrm{MnCoGeB}_{0.05}$ alloy at $1.5 \mathrm{~K}$. Figure $4(\mathrm{~b})$ shows the Rietveld refinement of the $\mathrm{ND}$ data of the $\mathrm{MnCoGeB}_{0.05}$ alloy collected at $1.5 \mathrm{~K}$. It should be noted that the lattice parameters of the orthorhombic structure were constrained by $c_{\text {ort }}=\sqrt{ } 3 b_{\text {ort }}$ in the refinements. The refinement results and a schematic representation of the magnetic structure are shown in Table I and Fig. 4(c), respectively. The Mn atoms are at the $4 c$ site, which splits into four positions: $(x, 1 / 4, z),(x+1 / 2,3 / 4, z+1 / 2)$, $(-x, 3 / 4,-z)$, and $(x+1 / 2,1 / 4,-z+1 / 2)$.The Mn moment is 2.20(3) $\mu_{B}$ and lies in the $a-c$ plane with a tilt angle $\varphi=19.4^{\circ}$ away from the $a$ axis at $1.5 \mathrm{~K}$. The $x$ components $\left(\mu_{x}\right)$ of the Mn moments at the four positions are equal to each other, while the $z$ components $\left(\mu_{z}\right)$ at the $(x, 1 / 4, z)$ and $(-x, 3 / 4,-z)$ positions are opposite to that at the $(x+1 / 2,3 / 4, z+1 / 2)$, and $(x+1 / 2,1 / 4,-z+1 / 2)$ positions. Therefore, the magnetic exchange interaction between the neighboring $\mathrm{Mn}$ atoms comprises both FM and antiferromagnetic (AFM) components. The Co atoms also occupy the $4 c$ site and carry a moment of $0.76(4) \mu_{B}$ parallel to the $a$ axis.

Additionally, as shown in the inset of Fig. 4(a), the intensity of the (001) peak decreases with rising temperature and eventually disappears at $T \geqslant 75 \mathrm{~K}$, while the intensity of the (100) peak is almost invariable between 1.5 and $75 \mathrm{~K}$. This suggests the disappearing of the AFM component of the Mn moment. At $T \geqslant 75 \mathrm{~K}$, the Mn moments are not canted and the ND patterns can be well fit with the Shubnikov magnetic space group $P 6_{3} / \mathrm{mm}^{\prime} \mathrm{c}^{\prime}$ (no. 194.270), derived from the parent hexagonal space group $P 6_{3} / m m c$ (no. 194) with a propagation vector $\boldsymbol{k}=(0,0,0)$. Figure 5(a) shows the Rietveld refinement of the ND data of $\mathrm{MnCoGeB}_{0.05}$ alloy collected at $75 \mathrm{~K}$. The corresponding magnetic structure is illustrated in Fig. 5(b). At $75 \mathrm{~K}$, the $\mathrm{MnCoGeB}_{0.05}$ alloy is in a hexagonal structure, where both $\mathrm{Mn}$ and Co moments are parallel to the $c$ axis. The $\mathrm{Mn}$ and Co moments derived from the refinement are 2.01(2) $\mu_{B}$ and $0.72(4) \mu_{B}$, respectively.

Consequently, the $\mathrm{MnCoGeB}_{0.05}$ alloy undergoes a PM to FM transition at $T_{C}=260 \mathrm{~K}$, where the hexagonal structure (space group $P 6_{3} / m m c$, no. 194) is retained. At $T<75 \mathrm{~K}$, an extra AFM component arises in the Mn moment, which
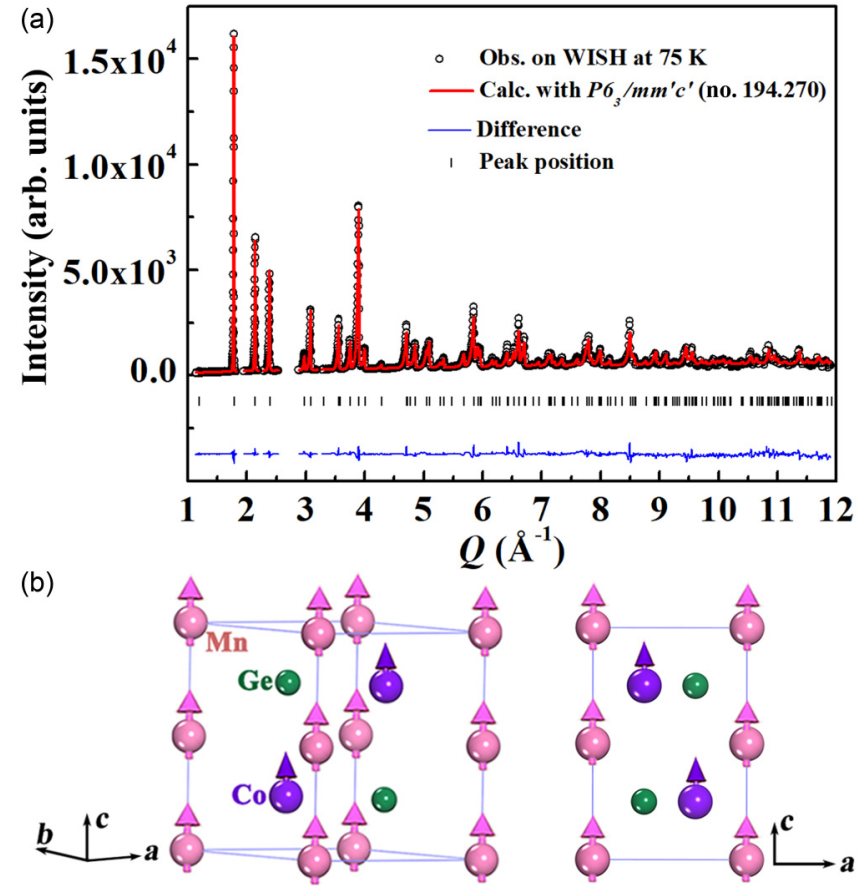

FIG. 5. (a) Rietveld refinement of the ND patterns at $T=75 \mathrm{~K}$ for the $\mathrm{MnCoGeB}_{0.05}$ alloy. Note that the nuclear and magnetic diffraction have the same peak positions. (b) Schematic representation of the magnetic structure in $\mathrm{MnCoGeB}_{0.05}$ alloy at $75 \mathrm{~K}$.

is perpendicular to the original FM component. This leads to a canted spin arrangement in the magnetic structure and thus breaks the hexagonal $P 6_{3} / \mathrm{mm}^{\prime} \mathrm{c}^{\prime}$ (no. 194.270) symmetry. As a result, the hexagonal $P 6_{3} / \mathrm{mm}^{\prime} \mathrm{c}^{\prime}$ (no. 194.270) symmetry is lowered to its distorted derivative, i.e., the orthorhombic $P n m^{\prime} a^{\prime}$ (no. 62.447). In strong contrast to a complete hexagonal-orthorhombic structural transition in the $\mathrm{MnCoGeB}_{0.01}$ alloy, the $\mathrm{MnCoGeB}_{0.05}$ alloy only shows subtle distortions of the hexagonal structure as evidenced by a canted spin arrangement, while the specific relationship between the lattice parameters (i.e., $c_{\text {ort }}=\sqrt{ } 3 b_{\text {ort }}$ ) is retained.

Apart from the difference in magnetic structure between the $\mathrm{MnCoGeB}_{0.05}$ and the $\mathrm{MnCoGe} / \mathrm{MnCoGeB}_{0.01}$ alloys, the size of the Mn moment also shows a substantial difference. The Mn atom carries a large moment of 3.54(3) $\mu_{B}$ and $3.08(4) \mu_{B}$ in the $\mathrm{MnCoGe}$ and $\mathrm{MnCoGeB}_{0.01}$ alloys, respectively, while the Mn moment is only $2.20(3) \mu_{B}$ in the $\mathrm{MnCoGeB}_{0.05}$ alloy. DFT calculations on the $\mathrm{Mn} M^{\prime}$ Ge alloys reveal that the size of the Mn moment strongly depends on the $\mathrm{Mn}-\mathrm{Mn}$ interatomic distances [44-46]. As shown in Table I, the Mn-Mn distances are 3.079(2), 3.064(3), and 2.629(1) for the $\mathrm{MnCoGe}, \mathrm{MnCoGeB}_{0.01}$, and $\mathrm{MnCoGeB}_{0.05}$ alloys, respectively. The significantly shortened $\mathrm{Mn}-\mathrm{Mn}$ distance in the $\mathrm{MnCoGeB}_{0.05}$ alloy may cause considerable overlap of the $3 d$ orbitals between the neighboring $\mathrm{Mn}$ atoms, which weakens the exchange splitting between the majority and minority $3 d$ bands and thus leads to a small $\mathrm{Mn}$ moment in the $\mathrm{MnCoGeB}_{0.05}$ alloy. In strong contrast to the significant reduction of the Mn moment upon boron doping, the size of the Co moment is almost constant, although the Co-Co distance is expanded from 2.813(3) to 3.519(3) $\AA$ as the boron 
content increases from $1 \%$ to $5 \%$. Previous studies [20,41] reveal that the Co atoms tend to form covalent bonding with the nearest $\mathrm{Ge}$ atoms due to the relatively short $\mathrm{Ge}-\mathrm{Co}$ interatomic distance $(\sim 2.33 \AA)$. The electron pairing due to the Co-Ge covalent bonding reduces the exchange splitting between the majority and minority $3 d$ bands of the Co atoms, leading to small Co moment (see Table I). Consequently, the size of the Co moment is predominantly determined by the Co-Ge covalent bonding. As shown in Table I, the Co-Ge distance is almost constant with increasing boron from $1 \%$ to $5 \%$, which leads to an invariable character of the Co moment.

\section{Stability of the hexagonal phase}

One can learn from the ND and magnetic results that the boron addition has a strong influence on the hexagonalorthorhombic structural transition temperature $T_{t}$. The $T_{t}$ is about $430 \mathrm{~K}$ upon cooling in the parent $\mathrm{MnCoGe}$ alloy, which is reduced to approximately $293 \mathrm{~K}$ in the $\mathrm{MnCoGeB}_{0.01}$ alloy. The hexagonal to orthorhombic transition is greatly suppressed in the $\mathrm{MnCoGeB}_{0.05}$ alloy, although slight lattice distortion occurs in the hexagonal structure below $75 \mathrm{~K}$. Therefore, the boron addition actually stabilizes the hightemperature hexagonal phase and thus shifts the $T_{t}$ to lower temperatures. When the $T_{t}$ is lowered to the temperature range $T_{C}{ }^{\text {hex }}<T_{t}<T_{C}{ }^{\text {ort }}$, a magnetostructural FOMT between the PM hexagonal and FM orthorhombic phases can be triggered, as demonstrated in the $\mathrm{MnCoGeB}_{0.01}$ alloy. The realization of the magnetostructural FOMT in the MnCoGe alloys via other ways (e.g., off-stoichiometry, element substitution) [15-23], can essentially be attributed to the same origin, i.e., via tailoring the stability of the hexagonal phase. However, the underlying mechanism for stabilizing the hexagonal structure in the MnCoGe alloys has not been well understood yet.

Previous studies on the Fe-substituted MnNiGe alloys, belonging to the same $\mathrm{Mn} M^{\prime} \mathrm{Ge}$ family as the $\mathrm{MnCoGe}$ alloys, suggest that the stabilization of the hexagonal structure originates from the strengthening of the covalent bonding between the neighboring atoms [38]. Since covalent bonding is sensitive to interatomic distances, we first examine the dependence of interatomic distances on the boron addition for the hexagonal MnCoGe alloys. Note that the hexagonal structure appears in the parent MnCoGe alloy above $650 \mathrm{~K}$ upon heating from room temperature [10], beyond our ND measurement temperature range. As a result, Fig. 6 only shows the temperature-dependent interatomic distances for the $\mathrm{MnCoGeB}_{0.01}$ and $\mathrm{MnCoGeB}_{0.05}$ alloys in the hexagonal structure. Obviously, boron addition shortens the nearest interatomic distances in the hexagonal structure, which is expected to strengthen the covalent bonding and hence stabilize the hexagonal phase.

To get further insight into the influence of boron addition on the covalent bonding in the hexagonal structure, we performed valence-electron localization function (ELF) calculations [33] for the hexagonal MnCoGe alloys (see the Sec. III for details). Higher ELF values correspond to more localized electrons, suggesting a stronger covalent bonding between the neighboring atoms.

Figure 7 compares the ELF contour map in the (110) plane of the hexagonal structure for the $\mathrm{MnCoGeB}_{0.01}$ and
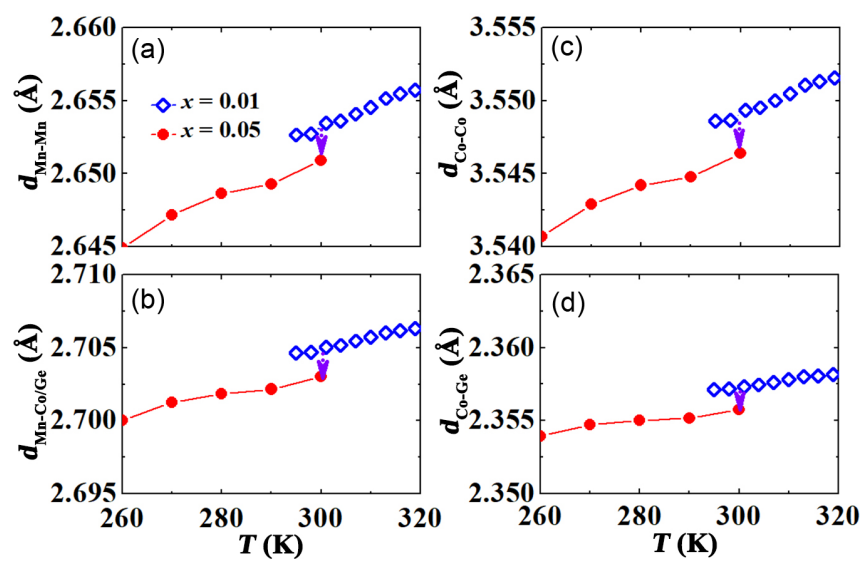

FIG. 6. Thermal evolution of the nearest interatomic distances for the $\mathrm{MnCoGeB}_{x}$ alloys in the hexagonal structure, $x=0.01$ (open blue symbols) and 0.05 (solid red symbols). The errors on the refined distances are smaller than the symbol sizes.

$\mathrm{MnCoGeB}_{0.05}$ alloys. In both alloys, strong electron localization can be observed around the $\mathrm{Ge}$ atoms. In order to quantitatively study the subtle variations in covalent bonding between the $\mathrm{MnCoGeB}_{0.01}$ and $\mathrm{MnCoGeB}_{0.05}$ alloys, Figs. 7(c)-7(f) plot the line profiles of ELF values between the nearest-neighboring atoms. Maximum ELF values reach approximately 0.49 and 0.56 for the nearest Ge-Mn and GeCo atoms, respectively, indicating strong covalent bonding between the nearest Ge-Mn and Ge-Co atoms. The stronger covalent bonding in the latter may be ascribed to the shorter Ge-Co interatomic distances $[\sim 2.36 \AA$ shown in Fig. 6(d)] compared with the Ge-Mn interatomic distances $[\sim 2.70 \AA$ shown in Fig. 6(b)]. In contrast to the strong electron localization between the nearest Ge-Mn and Ge-Co atoms, weak electron localization has be observed for the nearest Mn-Mn and Co-Co atoms. With an increase in the boron content from 0.01 to 0.05 , the ELF value of the nearest $\mathrm{Mn}-\mathrm{Mn}$ atoms is increased by about $3.95 \%$ [see inset of Fig. 7(e)], in line with the shortened Mn-Mn distances [see Fig. 6(a)], while the ELF values of other nearest-neighboring atoms are almost invariant. This reveals a strong dependence of the Mn-Mn covalent bonding on the nearest $\mathrm{Mn}-\mathrm{Mn}$ distances. Consequently, boron addition shortens the interatomic distances and strengthens the covalent bonding between the neighboring $\mathrm{Mn}-\mathrm{Mn}$ atoms, which stabilizes the high-temperature hexagonal phase (i.e., lowers the $T_{t}$ ) and thus triggers the magnetostructural FOMT in the MnCoGe alloys.

\section{Structural evolution at the magnetostructural transition}

Figure 8 presents the thermal evolution of the unit-cell parameters derived from the ND experiments for the $\mathrm{MnCoGe}$ and $\mathrm{MnCoGeB}_{0.05}$ alloys, respectively. Both samples show continuous changes in the unit-cell parameters at the FM transition.

In strong contrast to the continuous structure variations in the $\mathrm{MnCoGe}$ and $\mathrm{MnCoGeB}_{0.05}$ alloys, a significant lattice discontinuity occurs at the magnetostructural FOMT of the $\mathrm{MnCoGeB}_{0.01}$ alloy (see Fig. 9). As depicted in the inset of Fig. 9(a), the unit-cell parameters of the orthorhombic 

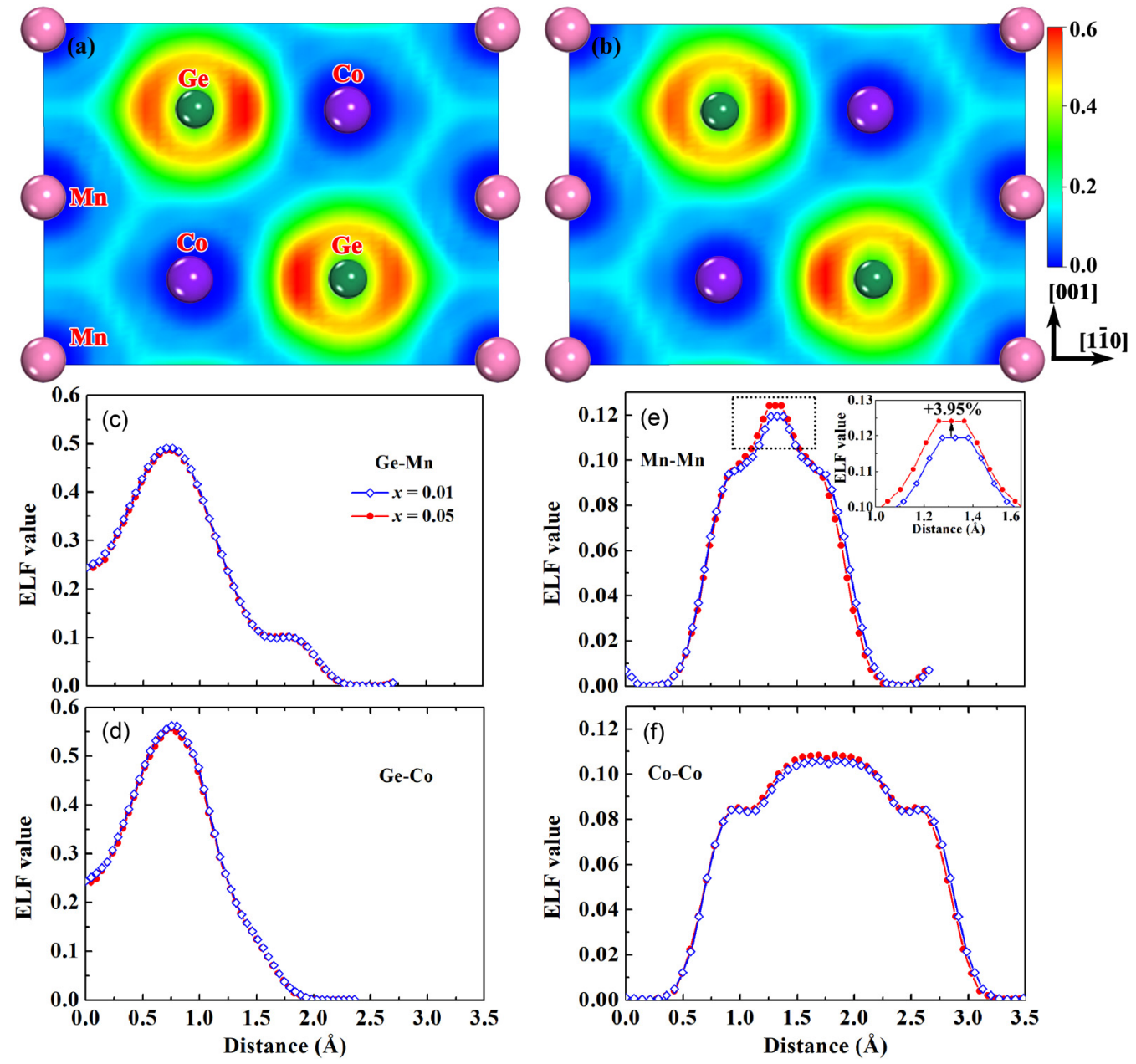

FIG. 7. ELF contour map in the (110) plane of the hexagonal MnCoGeB $x$ alloys with $x=0.01$ (a) and 0.05 (b). Line profile of the ELF values between the nearest Ge-Mn (c), Ge-Co (d), Mn-Mn (e), and Co-Co (f) atoms for both $x=0.01$ and 0.05 alloys.
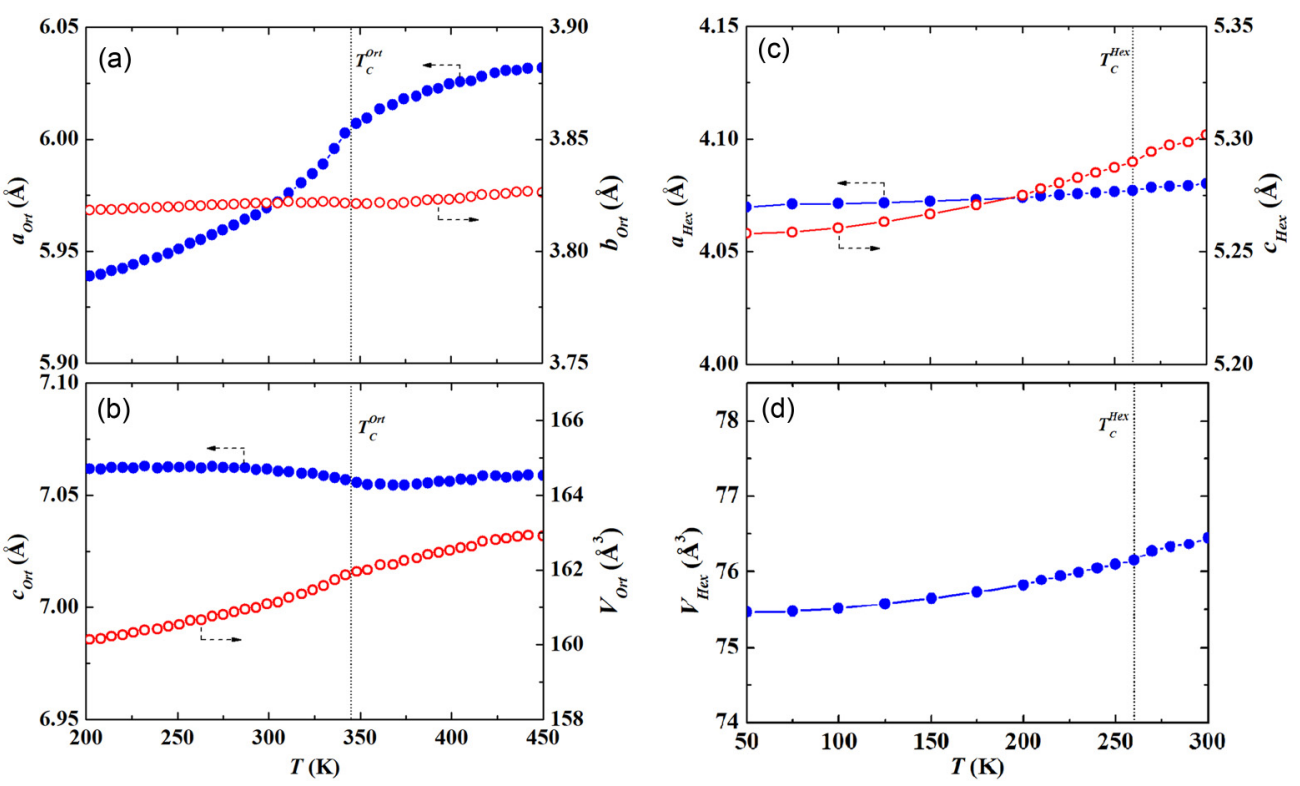

FIG. 8. Thermal evolution of unit-cell parameters for the orthorhombic MnCoGe (a), (b) and hexagonal $\mathrm{MnCoGeB}_{0.05}$ (c), (d) alloys. The black dotted lines in the panels represent the Curie temperature. The errors on the refined unit-cell parameters are smaller than the symbol size. 

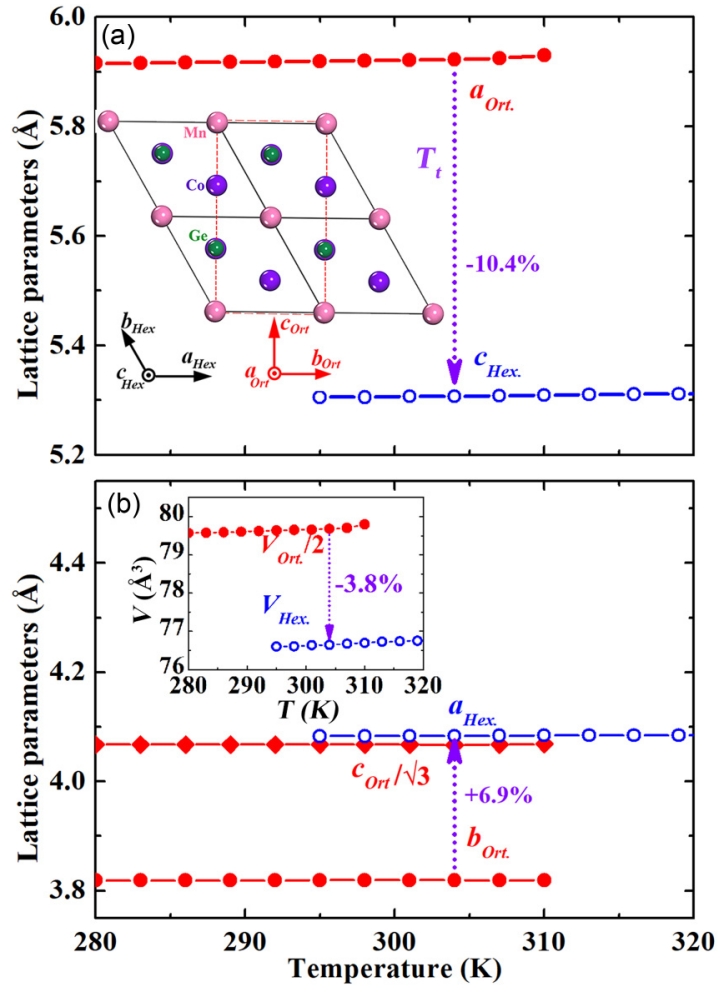

FIG. 9. Temperature-dependent unit-cell parameters for the $\mathrm{MnCoGeB}_{0.01}$ alloy. The solid red and open blue symbols represent the lattice parameters for the orthorhombic and hexagonal phases, respectively. The crystallographic relationship between the hexagonal (solid black lines) and orthorhombic (dashed red lines) phases is illustrated in the inset of (a). The dotted arrows represent the magnetostructural transition temperature. The errors on the refined unit-cell parameters are smaller than the symbol size.

and hexagonal structures are related by $a_{\text {ort }}=c_{\text {hex }}, b_{\text {ort }}=$ $a_{\text {hex }}, c_{\text {ort }}=\sqrt{ } 3 a_{\text {hex }}$, and $V_{\text {ort }}=2 V_{\text {hex }}$. The orthorhombic lattice shrinks by $10.4 \%$ along the $a$ axis at the magnetostructural FOMT, while it expands by $6.9 \%$ and $0.4 \%$ along the $b$ and $c$ axes, respectively (see Fig. 9). The anisotropic changes in the unit-cell dimensions finally give rise to a volume contraction of about 3.8\% upon the orthorhombic-hexagonal transition [see the inset of Fig. 9(b)]. The striking crystallographic changes lead to a remarkable structural entropy change, which can account for over $90 \%$ of the total entropy change for the MnCoGe-based magnetocaloric alloys [47].

The changes in lattice parameters reflect the variations in the interatomic distances, which strongly influence the magnetic exchange interaction and chemical bonding in the MnCoGe-based alloys. Figure 10 presents the temperature dependence of the nearest interatomic distances in the $\mathrm{MnCoGeB}_{0.01}$ alloy. The nearest $\mathrm{Mn}-\mathrm{Mn}$ distance is strongly reduced during the orthorhombic-hexagonal transition, while the Co-Co distance is significantly increased. In the meantime, small variations in the $\mathrm{Mn}-\mathrm{Co} / \mathrm{Ge}$ and $\mathrm{Co}-\mathrm{Ge}$ distances are observed. Consequently, the high-temperature hexagonal phase is marked by a smaller Mn-Mn distance and a larger Co-Co distance than the low-temperature orthorhombic phase. As mentioned above, the size of the Mn moment critically depends on the Mn-Mn interatomic distance. The remarkable
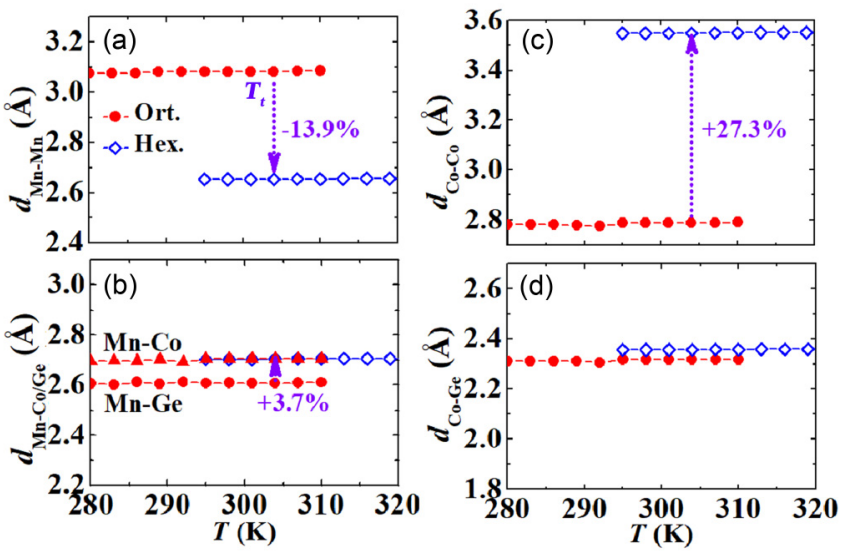

FIG. 10. Thermal evolution of interatomic distances for the $\mathrm{MnCoGeB}_{0.01}$ alloy. The solid red and open blue symbols represent the interatomic distances for the orthorhombic and hexagonal phases, respectively. The dotted arrows represent the magnetostructural transition temperature. The errors on the refined distances are smaller than the symbol size.

decrease in the Mn-Mn distance during the FOMT transition may result in not only an order-disorder transition of the moment arrangement, but also a partial quenching of the $\mathrm{Mn}$ moment. A similar phenomenon has been observed in the FOMT of $\mathrm{FeRh}$ [48], $(\mathrm{Mn}, \mathrm{Fe})_{2}(\mathrm{P}, \mathrm{Si})$ [49,50], $\mathrm{La}(\mathrm{Fe}, \mathrm{Si})$ [51], and $\mathrm{Eu}_{2} \mathrm{In}$ [52] alloys. DFT calculations [53] and ND experiments [48] reveal that the FOMT in the FeRh alloys is accompanied with an instability of the $\mathrm{Rh}$ moment due to considerable changes in the interatomic distances and an electronic redistribution. Our previous DFT calculations [50] and synchrotron x-ray-diffraction/-absorption experiments [49] indicate that the $(\mathrm{Mn}, \mathrm{Fe})_{2}(\mathrm{P}, \mathrm{Si})$ compounds experience a partial quenching of the Fe moment, as well as a strong electronic redistribution around the $\mathrm{Fe}$ atoms during the FOMT. Similarly, the DFT studies on the $\mathrm{La}(\mathrm{Fe}, \mathrm{Si})_{13}$ compounds suggest an instability of the Fe moment during the FOMT [51]. A recent report on the $\mathrm{Eu}_{2} \mathrm{In}$ rare-earth intermetallic compound also demonstrates that the observed FOMT is due to an instability of the Eu moment [52]. Consequently, further studies to monitor the electronic redistribution around the $\mathrm{Mn}$ atoms (e.g., by DFT and synchrotron x-ray diffraction), as well as the evolution of the Mn moment during the FOMT (e.g., by ND and x-ray magnetic circular dichroism), will provide more insight into the instability of the Mn moment and the phase-transition mechanism for the $\mathrm{MnM} / \mathrm{Ge}$ alloys.

\section{CONCLUSIONS}

In summary, we have studied the phase-transition behavior and magnetic structure of the $\mathrm{MnCoGeB}_{x}(x=0$, 0.01 , and 0.05 ) alloys by temperature-dependent neutrondiffraction and density functional theory calculations. It is found that boron addition offers an efficient and effective way to manipulate the magnetostructural coupling in the $\mathrm{MnCoGe}$ alloys. The originally separated magnetic and structural transitions in the MnCoGe alloy were tailored to coincide after 1 at. \% boron addition. With a further increase in the boron addition to 5 at. \%, the magnetic and structural transitions 
were decoupled again due to the significantly suppressed hexagonal-orthorhombic structural transition. The tuning effect of boron addition can be attributed to the decrease in the interatomic distances, which strengthens the covalent bonding and enhances the stability of the high-temperature hexagonal phase. The $\mathrm{MnCoGe}$ and $\mathrm{MnCoGeB}_{0.01}$ alloys shows a collinear FM structure below $T_{C}$, where both the $\mathrm{Mn}$ and Co moments are parallel to the $c$ axis of the orthorhombic structure. The $\mathrm{MnCoGeB}_{0.05}$ alloy undergoes a PM-FM transition at $T_{C}^{\text {hex }}=260 \mathrm{~K}$, where both the Mn and Co moments starts to align along to the $c$ axis of the hexagonal structure. Below $75 \mathrm{~K}$, an additional AFM component arises in the Mn moment along the $a$ axis, which leads to a canted spin arrangement and thus breaks the hexagonal symmetry. As a result, the hexagonal $P 6_{3} / \mathrm{mm}^{\prime} \mathrm{c}^{\prime}$ (no. 194.270) symmetry is lowered to its distorted derivative, i.e., the orthorhombic $P n m^{\prime} a^{\prime}$ (no. 62.447) symmetry. Apart from the different magnetic structures, the Mn moment in the $\mathrm{MnCoGeB}_{0.05}$ alloys is much smaller than that in the $\mathrm{MnCoGe}$ and $\mathrm{MnCoGeB}_{0.01}$ alloys, which is due to the shorter $\mathrm{Mn}-\mathrm{Mn}$ distance and the resultant weaker exchange splitting between the majority and minority $3 d$ bands. Additionally, in contrast to conventional second-order magnetic transition, the magnetostructural
FOMT transition in the $\mathrm{MnCoGeB}_{0.01}$ is accompanied with significant changes in the Mn-Mn distance that critically influences the size of the Mn moment. This suggests that the magnetostructural transition in the MnCoGe-based alloys may be characteristic of not only an order-disorder transition of the moment arrangement but also a sizable Mn moment. Consequently, our work demonstrates a strong coupling between the lattice, spin, and electron degrees of freedom in the $\mathrm{MnCoGe}-$ based alloys.

\section{ACKNOWLEDGMENTS}

X.M. acknowledges financial support from the National Natural Science Foundation of China (Grants No. U1832191 and No. 51801102) and the Natural Science Foundation of Jiangsu Province (Grants No. BK20180491 and No. BK20180418). This work is based on ND experiments performed at the Swiss spallation neutron source SINQ, Paul Scherrer Institute, Villigen in Switzerland and at the ISIS Facility, Rutherford Appleton Laboratory in the UK. This work is part of the Industrial Partnership Program of the Dutch Foundation for Fundamental Research on Matter (FOM), and was cofinanced by BASF New Business.
[1] A. Kitanovski, Energy applications of magnetocaloric materials, Adv. Energy Mater. 10, 1903741 (2020).

[2] T. Gottschall, K. P. Skokov, M. Fries, A. Taubel, I. Radulov, F. Scheibel, D. Benke, S. Riegg, and O. Gutfleisch, Making a cool choice: the materials library of magnetic refrigeration, Adv. Energy Mater. 9, 1901322 (2019).

[3] V. Franco, J. S. Blázquez, B. Ingale, and A. Conde, The magnetocaloric effect and magnetic refrigeration near room temperature: materials and models, Annu. Rev. Mater. Res. 42, 305 (2012)

[4] P. Weiss and A. Piccard, Le phénomène magnétocalorique, J. Phys. Theor. Appl. 7, 103 (1917).

[5] X. F. Miao, L. Caron, P. Roy, N. H. Dung, L. Zhang, W. A. Kockelmann, R. A. de Groot, N. H. van Dijk, and E. Brück, Tuning the phase transition in transition-metal-based magnetocaloric compounds, Phys. Rev. B 89, 174429 (2014).

[6] X. F. Miao, Y. Mitsui, A. I. Dugulan, L. Caron, N. V. Thang, P. Manuel, K. Koyama, K. Takahashi, N. H. van Dijk, and E. Brück, Kinetic-arrest-induced phase coexistence and metastability in(Mn, Fe) $)_{2}(\mathrm{P}, \mathrm{Si})$, Phys. Rev. B 94, 094426 (2016).

[7] M. F. J. Boeije, M. Maschek, X. F. Miao, N. V. Thang, N. H. van Dijk, and E. Brück, Mixed magnetism in magnetocaloric materials with first-order and second-order magnetoelastic transitions, J. Phys. D: Appl. Phys. 50, 174002 (2017).

[8] F. X. Hu, B. G. Shen, J. R. Sun, Z. H. Cheng, G. H. Rao, and X. X. Zhang, Influence of negative lattice expansion and metamagnetic transition on magnetic entropy change in the compound $\mathrm{LaFe}_{11.4} \mathrm{Si}_{1.6}$, Appl. Phys. Lett. 78, 3675 (2001).

[9] A. Fujita, S. Fujieda, Y. Hasegawa, and K. Fukamichi, Itinerantelectron metamagnetic transition and large magnetocaloric effects in $\mathrm{La}\left(\mathrm{Fe}_{x} \mathrm{Si}_{1-x}\right)_{13}$ compounds and their hydrides, Phys. Rev. B 67, 104416 (2003).
[10] S. Nizioł, A. Wesełucha, W. Bażela, and A. Szytuła, Magnetic properties of the $\mathrm{Co}_{x} \mathrm{Ni}_{1-x} \mathrm{MnGe}$ system, Solid State Commun. 39, 1081 (1981).

[11] S. Kaprzyk and S. Nizioł, The electronic structure of CoMnGe with the hexagonal and orthorhombic crystal structure, J. Magn. Magn. Mater. 87, 267 (1990).

[12] Y. Li, Q. Zeng, Z. Wei, E. Liu, X. Han, Z. Du, L. Li, X. $\mathrm{Xi}$, W. Wang, and S. Wang, An efficient scheme to tailor the magnetostructural transitions by staged quenching and cyclical ageing in hexagonal martensitic alloys, Acta Mater. 174, 289 (2019).

[13] E. K. Liu, W. Zhu, L. Feng, J. L. Chen, W. H. Wang, G. H. Wu, H. Y. Liu, F. B. Meng, H. Z. Luo, and Y. X. Li, Vacancy-tuned paramagnetic/ferromagnetic martensitic transformation in $\mathrm{Mn}$ poor $\mathrm{Mn}_{1-x}$ CoGe alloys, Europhys. Lett. 91, 17003 (2010).

[14] S. C. Ma, Q. Ge, Y. F. Hu, L. Wang, K. Liu, Q. Z. Jiang, D. H. Wang, C. C. Hu, H. B. Huang, G. P. Cao, Z. C. Zhong, and Y. W. $\mathrm{Du}$, Driving higher magnetic field sensitivity of the martensitic transformation in MnCoGe ferromagnet, Appl. Phys. Lett. 111, 192406 (2017).

[15] S. C. Ma, Y. X. Zheng, H. C. Xuan, L. J. Shen, Q. Q. Cao, D. H. Wang, Z. C. Zhong, and Y. W. Du, Large room temperature magnetocaloric effect with negligible magnetic hysteresis losses in $\mathrm{Mn}_{1-x} \mathrm{~V}_{x}$ CoGe alloys, J. Magn. Magn. Mater. 324, 135 (2012).

[16] O. Tozkoparan, O. Yildirim, E. Yüzüak, E. Duman, and I. Dincer, Magnetostructural transition in Co-Mn-Ge systems tuned by valence electron concentration, J. Alloys Compd. 791, 208 (2019).

[17] N. T. Trung, V. Biharie, L. Zhang, L. Caron, K. H. J. Buschow, and E. Brück, Giant magnetocaloric effects by tailoring the phase transitions, Appl. Phys. Lett. 96, 162507 (2010). 
[18] G. J. Li, E. K. Liu, H. G. Zhang, Y. J. Zhang, J. L. Chen, W. H. Wang, H. W. Zhang, G. H. Wu, and S. Y. Yu, Phase diagram, ferromagnetic martensitic transformation and magnetoresponsive properties of Fe-doped MnCoGe alloys, J. Magn. Magn. Mater. 332, 146 (2013).

[19] E. Yüzüak, I. Dincer, Y. Elerman, I. Dumkow, B. Heger, and S. Yuce Emre, Enhancement of magnetocaloric effect in $\mathrm{CoMn}_{0.9} \mathrm{Fe}_{0.1} \mathrm{Ge}$ alloy, J. Alloys Compd. 641, 69 (2015).

[20] Q. Ren, W. D. Hutchison, J. Wang, A. J. Studer, and S. J. Campbell, Magnetic and structural transitions tuned through valence electron concentration in magnetocaloric $\mathrm{Mn}\left(\mathrm{Co}_{1-x} \mathrm{Ni}_{x}\right) \mathrm{Ge}$, Chem. Mater. 30, 1324 (2018).

[21] S. K. Pal, C. Frommen, S. Kumar, B. C. Hauback, H. Fjellvåg, T. G. Woodcock, K. Nielsch, and G. Helgesen, Comparative phase transformation and magnetocaloric effect study of Co and $\mathrm{Mn}$ substitution by $\mathrm{Cu}$ in $\mathrm{MnCoGe}$ compounds, J. Alloys Compd. 775, 22 (2019).

[22] R. R. Wu, L. F. Bao, F. X. Hu, H. Wu, Q. Z. Huang, J. Wang, X. L. Dong, G. N. Li, J. R. Sun, and F. R. Shen, Giant barocaloric effect in hexagonalNi $\mathrm{i}_{2} \mathrm{In}$-type $\mathrm{Mn}-\mathrm{Co}-\mathrm{Ge}-$ In compounds around room temperature, Sci. Rep. 5, 18027 (2015).

[23] Y. Li, H. Zhang, K. Tao, Y. Wang, M. Wu, and Y. Long, Giant magnetocaloric effect induced by reemergence of magnetostructural coupling in $\mathrm{Si}$-doped $\mathrm{Mn}_{0.95} \mathrm{CoGe}$ compounds, Mater. Des. 114, 410 (2017).

[24] S. Nizioł, A. Ziçba, R. Zach, M. Baj, and L. Dmowski, Structural and magnetic phase transitions in $\mathrm{Co}_{x} \mathrm{Ni}_{1-x} \mathrm{MnGe}$ system under pressure, J. Magn. Magn. Mater. 38, 205 (1983).

[25] S. Anzai and K. Ozawa, Coupled nature of magnetic and structural transition in MnNiGe under pressure, Phys. Rev. B 18, 2173 (1978).

[26] S. NizioŁ, R. Zach, J. P. Senateur, and J. Beille, Pressure dependence of the magnetic transition temperature of the $\mathrm{CoMnGe}_{1-x} \mathrm{Si}_{x}$ system, J. Magn. Magn. Mater. 79, 333 (1989).

[27] L. Caron, N. Trung, and E. Brück, Pressure-tuned magnetocaloric effect in $\mathrm{Mn}_{0.93} \mathrm{Cr}_{0.07} \mathrm{CoGe}$, Phys. Rev. B 84, 020414(R) (2011).

[28] P. Fischer, G. Frey, M. Koch, M. Könnecke, V. Pomjakushin, J. Schefer, R. Thut, N. Schlumpf, R. Bürge, and U. Greuter, High-resolution powder diffractometer HRPT for thermal neutrons at SINQ, Phys. B (Amsterdam, Neth.) 276-278, 146 (2000).

[29] L. C. Chapon, P. Manuel, P. G. Radaelli, C. Benson, L. Perrott, S. Ansell, N. J. Rhodes, D. Raspino, D. Duxbury, and E. Spill, Wish: the new powder and single crystal magnetic diffractometer on the second target station, Neutron News 22, 22 (2011).

[30] J. Rodriguez-Carvajal, Recent advances in magnetic structure determination by neutron powder diffraction, Phys. B (Amsterdam, Neth.) 192, 55 (1993).

[31] M. C. Payne, M. P. Teter, D. C. Allan, T. A. Arias, and J. D. Joannopoulos, Iterative minimization techniques for $a b$ initiototal-energy calculations: molecular dynamics and conjugate gradients, Rev. Mod. Phys. 64, 1045 (1992).

[32] J. P. Perdew, K. Burke, and M. Ernzerhof, Generalized Gradient Approximation Made Simple, Phys. Rev. Lett. 77, 3865 (1996).
[33] A. D. Becke and K. E. Edgecombe, A simple measure of electron localization in atomic and molecular systems, J. Chem. Phys. 92, 5397 (1990).

[34] B. Silvi and A. Savin, Classification of chemical bonds based on topological analysis of electron localization functions, Nature (London) 371, 683 (1994).

[35] J. Y. Raty, E. Schwegler, and S. A. Bonev, Electronic and structural transitions in dense liquid sodium, Nature (London) 449, 448 (2007).

[36] H. S. Rzepa, The importance of being bonded, Nat. Chem. 1, 510 (2009).

[37] M. Xu, Y. Q. Cheng, H. W. Sheng, and E. Ma, Nature of Atomic Bonding and Atomic Structure in the Phase-Change $\mathrm{Ge}_{2} \mathrm{Sb}_{2} \mathrm{Te}_{5}$ Glass, Phys. Rev. Lett. 103, 195502 (2009).

[38] E. K. Liu, W. H. Wang, L. Feng, W. Zhu, G. J. Li, J. Chen, H. W. Zhang, G. H. Wu, C. B. Jiang, and H. B. Xu, Stable magnetostructural coupling with tunable magnetoresponsive effects in hexagonal ferromagnets, Nat. Commun. 3, 873 (2012).

[39] J. M. Perez-Mato, S. V. Gallego, E. S. Tasci, L. Elcoro, G. de la Flor, and M. I. Aroyo, Symmetry-based computational tools for magnetic crystallography, Annu. Rev. Mater. Res. 45, 217 (2015).

[40] See Supplemental Material at http://link.aps.org/supplemental/ 10.1103/PhysRevMaterials.4.104407 for the details on the determination of the magnetic structures for the MnCoGeB $(x=$ $0,0.01$, and 0.05) alloys.

[41] A. SzytuŁa, A. T. Pedziwiatr, Z. Tomkowicz, and W. Bażela, Crystal and magnetic structure of CoMnGe, CoFeGe, FeMnGe and NiFeGe, J. Magn. Magn. Mater. 25, 176 (1981).

[42] W. Jeitschko, A high-temperature x-ray study of the displacive phase transition in MnCoGe, Acta Crystallogr. Sect. B 31, 1187 (1975).

[43] V. Johnson, Diffusionless orthorhombic to hexagonal transitions in ternary silicides and germanide, Inorg. Chem. 14, 1117 (1975).

[44] Z. Gercsi and K. G. Sandeman, Structurally driven metamagnetism in MnP and related Pnma compounds, Phys. Rev. B 81, 224426 (2010).

[45] K. R. Hahn, E. Assaf, A. Portavoce, S. Bertaina, and A. Charaï, Structural and composition effects on electronic and magnetic properties in thermoelectric $\mathrm{Mn}_{1-x-y} \mathrm{Co}_{1+x} \mathrm{Ge}_{1+y}$ materials, J. Phys. Chem. C 121, 26575 (2017).

[46] F. Guillou, F. Wilhelm, O. Tegus, and A. Rogalev, Microscopic mechanism of the giant magnetocaloric effect in MnCoGe alloys probed by x-ray magnetic circular dichroism, Appl. Phys. Lett. 108, 122405 (2016).

[47] K. A. J. Gschneidner, Y. Mudryk, and V. K. Pecharsky, On the nature of the magnetocaloric effect of the first-order magnetostructural transition, Scr. Mater. 67, 572 (2012).

[48] N. Kunitomi, M. Kohgi, and Y. Nakai, Diffuse scattering of neutrons in the antiferromagnetic phase of FeRh, Phys. Lett. A 37, 333 (1971).

[49] M. F. J. Boeije, P. Roy, F. Guillou, H. Yibole, X. F. Miao, L. Caron, D. Banerjee, N. H. van Dijk, R. A. de Groot, and E. Brück, Efficient room-temperature cooling with magnets, Chem. Mater. 28, 4901 (2016).

[50] N. H. Dung, Z. Q. Ou, L. Caron, L. Zhang, D. T. C. Thanh, G. A. de Wijs, R. A. de Groot, K. H. J. Buschow, and E. Brück, Mixed magnetism for refrigeration and energy conversion, Adv. Energy Mater. 1, 1215 (2011). 
[51] M. E. Gruner, W. Keune, B. Roldan Cuenya, C. Weis, J. Landers, S. I. Makarov, D. Klar, M. Y. Hu, E. E. Alp, and J. Zhao, Element-Resolved Thermodynamics of Magnetocaloric $\mathrm{LaFe}_{13-x} \mathrm{Si}_{x}$, Phys. Rev. Lett. 114, 057202 (2015).

[52] F. Guillou, A. K. Pathak, D. Paudyal, Y. Mudryk, F. Wilhelm, A. Rogalev, and V. K. Pecharsky, Non-hysteretic first-order phase transition with large latent heat and giant low-field magnetocaloric effect, Nat. Commun. 9, 2925 (2018).

[53] M. E. Gruner, E. Hoffmann, and P. Entel, Instability of the rhodium magnetic moment as the origin of the metamagnetic phase transition in $\alpha$-FeRh, Phys. Rev. B 67, 064415 (2003). 